\title{
Mass of nonrelativistic meson from leading twist distribution amplitudes.
}

\author{
V.V. Braguta ${ }^{1, *}$ \\ ${ }^{1}$ Institute for High Energy Physics, Protvino, Russia
}

\begin{abstract}
In this paper distribution amplitudes of pseudoscalar and vector nonrelativistic mesons are considered. Using equations of motion for the distribution amplitudes, it is derived relations which allow one to calculate the masses of nonrelativistic pseudoscalar and vector meson if the leading twist distribution amplitudes are known. These relations can be also rewritten as relations between the masses of nonrelativistic mesons and infinite series of QCD operators, what can be considered as an exact version of Gremm-Kapustin relation in NRQCD.
\end{abstract}

PACS numbers: 12.38.-t, 12.38.Bx, 13.66.Bc, 13.25.Gv

\section{INTRODUCTION}

Study of hard exclusive processes can lead to a better understanding of mechanisms of hadrons production and hardrons structure. The description of such processes is based on the factorization theorem [1, 2]. Within this theorem the amplitude of hard exclusive process can be separated into two parts. The first part is partons production at very small distances, which can be treated within perturbative QCD. The second part is hardronization of the partons at larger distances. This part contains information about nonperturbative dynamic of strong interaction. For hard exclusive processes it can be parameterized by process independent distribution amplitudes (DA), which can be considered as hadrons' wave functions at light-like separation between the partons in the hadron.

Recently, the leading twist (twist-2) DAs of nonrelativistic mesons have become the object of intensive study $[3,4,15,6,6,8,19,10,11,12,13]$. In papers [14, 15] it was derived relations between twist-2 and twist-3 DAs of light mesons. Unfortunately, these relations contain DAs of higher Fock states, for which one has very poor knowledge. For nonrelativistic mesons, there states are suppressed by higher powers of relative velocoty of quark-antiqaurk pair inside meson [16], what leads to simplification of these relations. In papers [17, 18] it was shown that if one ignores the contribution of higher Fock states, one can write the solution of equations of motion for twist-3 DAs as an infinite series in Gegenbauer polynomials. Actually, using equations of motion one can find exact formula for the twist-3 DAs of nonrelativistic mesons through the leading twist DAs. In this paper we apply this exact formula and derive a relation between the masses of pseudoscalar and vector nonrelativistic mesons and leading twist DAs of these mesons.

This paper is organized as follows. Next section is devoted to the derivation of the relation between mass and the leading twist DA of pseudoscalar nonrelativistic meson. In section three similar relation will be derived for the vector meson.

\section{PSEUDOSCALAR NONRELATIVISTIC MESONS.}

In this section the relation which allows one find the mass of pseudoscalar nonrelativistic meson if the leading twist DA of this meson is known will be derived. To do this one needs the following DAs

$$
\begin{array}{r}
\left\langle P(p)\left|\bar{Q}(z) \gamma_{\alpha} \gamma_{5}[z,-z] Q(-z)\right| 0\right\rangle_{\mu}=i f_{P} p_{\alpha} \int_{-1}^{1} d \xi e^{i(p z)} \varphi_{A}(\xi, \mu), \\
\left\langle P(p)\left|\bar{Q}(z) \gamma_{5}[z,-z] Q(-z)\right| 0\right\rangle_{\mu}=i f_{P} \frac{M_{P}^{2}}{2 M_{Q}(\mu)} \int_{-1}^{1} d \xi e^{i(p z)} \varphi_{P}(\xi, \mu),
\end{array}
$$

where $\mu$ is the scale at which the axial and pseudoscalar DAs $\varphi_{A}(\xi, \mu), \varphi_{P}(\xi, \mu)$ are defined, $M_{Q}(\mu)$ is the running mass of the heavy quark $Q, M_{P}$ is the mass of the pseudoscalar meson $P, \xi$ is the fraction of the whole momentum of the meson $P$ carried by quark-antiquark pair, $[z,-z]$ is a gluon string which makes the corresponding operators gauge invariant.

Now few comments are in order. The meson $P$ is assumed to be nonrelativistic. The width of DAs of nonrelativistic meson is of order of $\xi^{2} \sim v^{2}$, where $v$ is relative velocity of quark-antiquark pair in the meson $P[5]$. In the end point region $|\xi| \sim 1$ the motion of quark-antiquark pair is relativistic, so DAs in these regions must be strongly suppressed.

*Electronic address: braguta@mail.ru 
One can even assume that DAs of nonrelativistic meson are zero in the end point region. However, as was shown in paper [5] radiative corrections, which can be taken into account through the evolution equation for the DAs, lead to the nonzero effect of the DAs in the end point region. In this paper all DAs and all scale dependent quantities will be taken at the scale at which radiative corrections are not very important and below explicit scale dependence will be omitted. For instance, in paper [5] it was shown that for charmonia mesons the effect of the evolution of the DAs is negligible at scale $\mu \sim \mu_{0}=1.2 \mathrm{GeV}$. It should be noted that at this scale $\mu \sim \mu_{0}$ the $\operatorname{DAs} \varphi_{A}(\xi, \mu), \varphi_{P}(\xi, \mu)$ are equal to each other at the leading order approximation in relative velocity [5].

In paper [14] it was shown that applying equations of motion one can derive the following relations between the moments of the DAs $\varphi_{A}(\xi), \varphi_{P}(\xi)$

$$
\left\langle\xi^{n}\right\rangle_{P}=\delta_{n 0}+\frac{n-1}{n+1}\left(\left\langle\xi^{n-2}\right\rangle_{P}-r\left\langle\xi^{n-2}\right\rangle_{A}\right),
$$

where $r=4 M_{Q}^{2} / M_{\eta}^{2}$, the moments $\left\langle\xi^{n}\right\rangle_{A, P}$ are defined as

$$
\left\langle\xi^{n}\right\rangle_{A, P}=\int_{-1}^{1} d \xi \xi^{n} \varphi_{A, P}(\xi) .
$$

It should be noted that actually equations (2) contan also terms which describe the contribution of higher Fock state quark-antiquark-gluon. However, for the nonrelativistic mesons such states are suppressed by large power of small relative velocity $v$ of quark-antiquark pair [16]. For this reason in equations (2) and below such terms are disregarded.

Equations (2) can be easily solved. The solution is given by the following expression

$$
\varphi_{P}(\xi)=C+r \int_{-1}^{\xi} \frac{\varphi_{A}^{\prime}(t)}{1-t^{2}} d t
$$

where the constant $C$ can be determined from the normalization condition for the function $\varphi_{P}(\xi)$

$$
\int_{-1}^{1} d \xi \varphi_{P}(\xi)=1=2 C+r \int_{-1}^{1} d \xi \frac{1+\xi^{2}}{\left(1-\xi^{2}\right)^{2}} \varphi_{A}(\xi)
$$

Note that equation (4) unambiguously determines the twist-3 DA $\varphi_{P}(\xi)$ through the twist-2 DA $\varphi_{A}(\xi)$. Actually this can always be done if one disregards the contribution of higher Fock states. One more example which demonstrates the last statement will be given below for vector nonrelativisitc meson.

As was explained above the DA $\varphi_{P}(\xi)$ is zero when $\xi$ is sufficiently near to the end points $\xi \rightarrow \pm 1$. In order to obtain this behaviour when $\xi \rightarrow \pm 1$ for the function $\varphi_{P}(\xi)$ in equation (4) one must set $C=0$, since the integral in the right hand side of equation (4) is zero for $\xi \sim \pm 1$. If one puts $C=0$ to equation (5), one gets the formula

$$
\frac{M_{P}^{2}}{4 M_{Q}^{2}}=\int_{-1}^{1} d \xi \frac{1+\xi^{2}}{\left(1-\xi^{2}\right)^{2}} \varphi_{A}(\xi)
$$

The running quark mass $M_{Q}$ here is taken at the scale at which radiative correction can be disregarded. Formula (6) is one of the main results of this paper.

It is known that the moments $\left\langle\xi^{n}\right\rangle_{A}$ can be expressed through the QCD operators

$$
\left\langle P(p)\left|\bar{Q} \gamma_{\nu} \gamma_{5}\left(i z^{\sigma} \stackrel{\leftrightarrow}{D}_{\sigma}\right)^{n} Q\right| 0\right\rangle=i f_{P} p_{\nu}(z p)^{n}\left\langle\xi^{n}\right\rangle_{A}
$$

Here

$$
\stackrel{\leftrightarrow}{D}=\vec{D}-\overleftarrow{D}, \quad \vec{D}=\vec{\partial}-i g B^{a}\left(\lambda^{a} / 2\right)
$$

From this perspective, equation (6) is exact expansion of the mass of the pseudoscalar meson $P$ in a infinite series of QCD operators $\left\langle\xi^{n}\right\rangle \sim\left\langle P(p)\left|\bar{Q} \gamma_{\nu} \gamma_{5}\left(i z^{\sigma} \stackrel{\leftrightarrow}{D}_{\sigma}\right)^{n} Q\right| 0\right\rangle$

$$
\frac{M_{P}^{2}}{4 M_{Q}^{2}}=\sum_{n=0}^{\infty}(n+1)\left\langle\xi^{n}\right\rangle=1+3\left\langle\xi^{2}\right\rangle_{A}+5\left\langle\xi^{4}\right\rangle_{A}+7\left\langle\xi^{6}\right\rangle_{A}+O\left(\left\langle\xi^{8}\right\rangle_{A}\right) .
$$

Recall that equation (6) is valid at the scale when radiative corrections are not very important. 
Formula (9) has very simple interpretation. To understand what does it mean let us recall that the moments $\left\langle\xi^{n}\right\rangle_{A}$ has the meaning of the average of the n-th power relative velocity of quark-antiquark pair in the direction of meson motion $\left\langle v_{z}^{n}\right\rangle[5]^{1}$. It is not difficult to understand that to get the average of the n-th power relative velocity $\left\langle v^{n}\right\rangle$, the $\left\langle v_{z}^{n}\right\rangle$ must be multiplied by a factor of $n+1$, since to get average one should take the integral $\sim d \cos \theta \cos ^{n} \theta$. So, one has the series $1+\left\langle v^{2}\right\rangle+\left\langle v^{4}\right\rangle+\left\langle v^{6}\right\rangle+\ldots$, which can be symbolically written as

$$
\frac{M_{P}^{2}}{4 M_{Q}^{2}}=\left\langle P(p)\left|\frac{1}{1-\hat{v}^{2}}\right| 0\right\rangle .
$$

Evidently, this is quantum expression for the energy $E=2 M / \sqrt{1-v^{2}}$ of two relativistic particle with zero net momentum moving with the speed $\hat{v}$.

In papers [17, 18, 19] it was pointed to the duality between light cone formalism and NRQCD description of hard exclusive processes. Although it was proved for some exclusive processes, today there is no strict proof of this duality. It would be interesting to know if it is possible to find formula in NRQCD dual to formula (6). To do this let us recall that in NRQCD one expands the mass of the pseudoscalar meson in operators

$$
\left\langle v^{n}\right\rangle=\frac{1}{M_{Q}^{2}} \frac{\left\langle P(p)\left|\chi^{+}(\overleftrightarrow{\mathbf{D}})^{n} \varphi\right| 0\right\rangle}{\left\langle P(p)\left|\chi^{+} \varphi\right| 0\right\rangle}
$$

where $\varphi$ and $\chi$ are bispinors which create antiquark and destroy quark respectively. To the leading order approximation one has $3\left\langle\xi^{2}\right\rangle=\left\langle v^{2}\right\rangle[5]$. If one puts this equation to (9]) one gets

$$
\frac{M_{P}^{2}}{4 M_{Q}^{2}}=1+\left\langle v^{2}\right\rangle+O\left(\left\langle v^{4}\right\rangle\right) .
$$

which is well known Gremm-Kapustin relation [20]. So, formulas (6), (9) can be considered as an exact version of Gremm-Kapustin relation.

Equation (6) can be used to test models of DAs. As an example let us take the models of $1 \mathrm{~S}$ and $2 \mathrm{~S}$ charmonia mesons proposed in papers [5, 7] at the central values of the parameters of these models. For the running mass of the c-quark $M_{c}$ the value $M_{c}=M_{c}^{\overline{M S}}\left(\mu=M_{c}^{\overline{M S}}\right)=1.23$ will be taken [21]. Thus one gets $M_{\eta_{c}}=2.81 \mathrm{GeV}, M_{\eta_{c}^{\prime}}=3.53$ $\mathrm{GeV}$, which is rather good estimation.

\section{VECTOR NONRELATIVISTIC MESONS.}

In this section the formula similar to formula (6) but for the vector nonrelativistic meson will be derived. To do this one needs the following DAs

$$
\begin{array}{r}
\left\langle V(p, \epsilon)\left|\bar{Q}_{\alpha}(z) Q_{\beta}(-z)\right| 0\right\rangle=\frac{f_{V} M_{V}}{4} \int_{0}^{1} d \xi e^{i(p z) \xi}\left\{\left(\hat{\epsilon}-\hat{p} \frac{(\epsilon z)}{(p z)}\right) \varphi_{\perp}(\xi)+\hat{p} \frac{(\epsilon z)}{(p z)} \varphi_{L}(\xi)+\right. \\
\left.\frac{f_{T}}{f_{V}} \frac{1}{M_{V}} \sigma_{\mu \nu} \epsilon^{\mu} p^{\nu} \varphi_{T}(\xi,)+\frac{1}{8}\left(1-\frac{f_{T}}{f_{V}} \frac{2 M_{Q}}{M_{V}}\right) e_{\mu \nu \sigma \rho} \gamma^{\mu} \gamma_{5} \epsilon^{\nu} p^{\sigma} z^{\rho} \varphi_{A}(\xi)\right\}_{\beta \alpha}
\end{array}
$$

where the constants $f_{T}, f_{V}$ are defined as

$$
\left\langle V(p, \epsilon)\left|\bar{Q} \gamma_{\alpha} Q\right| 0\right\rangle=f_{V} M_{V} \epsilon_{\alpha}, \quad\left\langle V(p, \epsilon)\left|\bar{Q} \sigma_{\alpha \beta} Q\right| 0\right\rangle=i f_{T}\left(p_{\alpha} \epsilon_{\beta}-p_{\beta} \epsilon_{\alpha}\right)
$$

The DAs $\varphi_{L}(\xi), \varphi_{T}(\xi)$ are twist-2 DAs, the DAs $\varphi_{\perp}(\xi), \varphi_{A}(\xi)$ are twist-3 DAs. The equations of motion for DAs (13) give [15]

$$
\begin{array}{r}
(n+1)\left\langle\xi^{n}\right\rangle_{\perp}=\left\langle\xi^{n}\right\rangle_{L}+\frac{n(n-1)}{2}(1-\delta)\left\langle\xi^{n-2}\right\rangle_{A}, \\
\frac{1}{2}(n+2)(1-\delta)\left\langle\xi^{n}\right\rangle_{A}=\left\langle\xi^{n}\right\rangle_{\perp}-\delta\left\langle\xi^{n}\right\rangle_{T}
\end{array}
$$

\footnotetext{
${ }^{1}$ It is assumed that the meson is moving along $z$-direction.
} 
where $\left\langle\xi^{n}\right\rangle_{L, T, \perp, A}$ are the moments of the DAs $\varphi_{L}(\xi), \varphi_{T}(\xi), \varphi_{\perp}(\xi), \varphi_{A}(\xi), \delta(\mu)=2 f_{T} / f_{V} M_{Q} / M_{V}$.

Equations (16) can be exactly solved [15]. For instance, the solution for the DA $\varphi_{A}$ is

$$
\varphi_{A}(\xi)=C_{0}+C_{1} \xi+\frac{1}{1-\delta}\left((1+\xi) \int_{\xi}^{1} \frac{\psi(t)}{1+t} d t+(1-\xi) \int_{-1}^{\xi} \frac{\psi(t)}{1-t} d t\right)
$$

where $\psi(\xi)=\varphi_{L}(\xi)+\delta \xi \varphi_{T}^{\prime}(\xi)$. The function $\varphi_{A}(\xi)$ must be even, normalized and it must be suppressed in the end point region. These requirements are fulfilled if $C_{0}=C_{1}=0$ and $\delta$ is equal to

$$
\frac{f_{T}}{f_{V}} \frac{2 M_{Q}}{M_{V}}=\frac{\int_{-1}^{1} \frac{d \xi}{1-\xi^{2}} \varphi_{L}(\xi)}{\int_{-1}^{1} d \xi \frac{1+\xi^{2}}{\left(1-\xi^{2}\right)^{2}} \varphi_{T}(\xi)}
$$

This formula is the analog of equation (6) but for vector meson $V$. It is more complicated than equation (6) since vector meson has two independent constants $f_{T}$ and $f_{V}$. Note that if one expands now right hand side of the last equation and puts Gremm-Kapustin relation (12) one will get NRQCD expression for the ratio $f_{T} / f_{V}[22$, 23]

$$
\frac{f_{T}}{f_{V}}=1-\frac{\left\langle v^{2}\right\rangle}{6}
$$

\section{Acknowledgments}

The author thanks A.K. Likhoded, A.V. Luchinsky for useful discussion. This work was partially supported by Russian Foundation of Basic Research under grant 07-02-00417, by president grant MK-140.2009.2 and scientific school grant SS-679.2008.2.

[1] G. P. Lepage and S. J. Brodsky, Phys. Rev. D 22, 2157 (1980).

[2] V. L. Chernyak and A. R. Zhitnitsky, Phys. Rept. 112, 173 (1984).

[3] G. T. Bodwin, D. Kang and J. Lee, Phys. Rev. D 74, 114028 (2006) arXiv:hep-ph/0603185.

[4] J. P. Ma and Z. G. Si, Phys. Lett. B 647, 419 (2007) arXiv:hep-ph/0608221.

[5] V. V. Braguta, A. K. Likhoded and A. V. Luchinsky, Phys. Lett. B 646, 80 (2007) arXiv:hep-ph/0611021.

[6] V. V. Braguta, Phys. Rev. D 75, 094016 (2007) arXiv:hep-ph/0701234.

[7] V. V. Braguta, Phys. Rev. D 77, 034026 (2008) arXiv:0709.3885 [hep-ph]].

[8] H. M. Choi and C. R. Ji, Phys. Rev. D 76, 094010 (2007) arXiv:0707.1173 [hep-ph]].

[9] T. Feldmann and G. Bell, AIP Conf. Proc. 964, 110 (2007) arXiv:0711.4014 [hep-ph]].

[10] G. Bell and T. Feldmann, JHEP 0804, 061 (2008) arXiv:0802.2221 [hep-ph]].

[11] V. V. Braguta, A. K. Likhoded and A. V. Luchinsky, Phys. Rev. D 79, 074004 (2009) [arXiv:0810.3607 [hep-ph]].

[12] C. W. Hwang, Eur. Phys. J. C 62, 499 (2009) arXiv:0811.0648 [hep-ph]].

[13] C. W. Hwang, JHEP 0910, 074 (2009) arXiv:0906.4412 [hep-ph]].

[14] P. Ball, JHEP 9901, 010 (1999) arXiv:hep-ph/9812375].

[15] P. Ball, V. M. Braun, Y. Koike and K. Tanaka, Nucl. Phys. B 529, 323 (1998) arXiv:hep-ph/9802299.

[16] G. T. Bodwin, E. Braaten and G. P. Lepage, Phys. Rev. D 51, 1125 (1995) [Erratum-ibid. D 55, 5853 (1997)] arXiv:hep-ph/9407339.

[17] V. V. Braguta, PoS C ONFINEMENT8, 097 (2008) [Phys. Rev. D 79, 074018 (2009)] arXiv:0811.2640 [hep-ph]].

[18] V. V. Braguta and V. G. Kartvelishvili, arXiv:0907.2772 [hep-ph].

[19] V. V. Braguta, A. K. Likhoded and A. V. Luchinsky, Phys. Rev. D 80, 094008 (2009) arXiv:0902.0459 [hep-ph]].

[20] M. Gremm and A. Kapustin, Phys. Lett. B 407, 323 (1997) arXiv:hep-ph/9701353.

[21] S. Narison, Phys. Lett. B 341, 73 (1994) arXiv:hep-ph/9408376.

[22] E. Braaten and Y. Q. Chen, Phys. Rev. D 57, 4236 (1998) [Erratum-ibid. D 59, 079901 (1999)] arXiv:hep-ph/9710357.

[23] V. V. Braguta, Phys. Rev. D 78, 054025 (2008) arXiv:0712.1475 [hep-ph]]. 